\title{
Perceived Needs of Parents of Critically Ill Children
}

Linda D. Scott

PURPOSE. To identify the needs of parents of critically ill hospitalized children as perceived by the parents and critical care nurses, and to identify any differences between the two groups. DESIGN. Descriptive, comparative.

SETTING. Pediatric intensive care unit (PICU) in a Midwestern hospital.

PARTICIPANTS. A nonprobability convenience sample of parents or primary caregivers of critically ill children $(\mathrm{n}=21)$ and pediatric critical care nurses $(\mathrm{n}=17)$.

outcome Measures. Critical Care Family Needs Inventory modified for pediatrics and demographic questionnaires. RESuLrs. Information, assurance, and proximity to the critically ill child were identified as priority needs of the PCGs in this study. Significant differences on specific needs were identified between PCG/nurse matched pairs; however, no significant differences were found in total scores between the two groups by a two-tailed paired $\mathrm{t}$ test. conclusions. This study supports the need to investigate interventions to better address parental needs of critically ill children. By consistent identification, prioritization, and incorporation of parental needs into the plan of care, nurses can assist the parents in the recognition and fulfillment of needs that have less perceived importance. Research-based interventions will facilitate improved parental adaptation to their child's critical hospitalization. Key words: Critical care nurses, critical hospitalizations, critically ill children, parental needs
Linda D. Scott, MSN, RN, is an adjunct faculty member, Grand Valley State University, Allendale, MI, and a doctoral student, University of Michigan, Ann Arbor.

$\mathbf{T}_{\mathrm{r}}$ he unexpected hospitalization of a child with a critical illness can be overwhelming and stressful for parents and their children. Frequently, during the critical periods of hemodynamic stabilization of the child, the needs of parents are considered insignificant by nurses. Once the critical period is resolved, attention may be directed toward the parents, yet their specific needs may remain unidentified and unfulfilled. Often, it is critical care nurses who identify and determine which parental needs will be addressed during the hospitalization of a child (Kleinpell \& Powers, 1992). However, parental needs as perceived by parents and nurses may be incongruent. When perceived parental needs are not accurately identified or addressed, the combination of incongruencies and the significant stress of having a critically ill child can result in parental anxieties escalating to a crisis event. Additionally, parental anxieties may be conveyed to the child and hinder recovery (Turner, Tomlinson, \& Harbaugh, 1990).

In order to facilitate family-centered pediatric critical care nursing, the parental needs as perceived by the parents must be accurately identified, prioritized, and incorporated into the plan of care for the family. Furthermore, accurate identification and prioritization of parental needs are pivotal in the development of effective nursing interventions that will maximize coping strategies and facilitate parental adaptation during the hospitalization of a critically ill child (Graves \& Hayes, 1996; LaMontagne \& Pawlak, 1990). This study was initiated to identify the needs of parents of critically ill hospitalized children as perceived by the parents and critical care nurses and to examine the differences between them in order to ascertain the accuracy of nursing assessments. 


\section{Review of the Literature}

There has been an increased awareness of the stressors experienced by family members of critically ill patients. Empirical research has identified sources of stress related to psychosocial factors and the physical environment (Carter, Miles, Buford, \& Hassanein, 1985; Miles, Carter, Spicher, \& Hassanein, 1984), changes in parental role (Carter et al.; Heuer, 1993; Jay \& Youngblut, 1991), parental uncertainty (LaMontagne, Johnson, \& Hepworth, 1995; Mishel, 1983; Tomlinson, Kirschbaum, Harbaugh, \& Anderson, 1996), and separation from family members (Philichi, 1989). According to Carter et al., emotional and behavioral responses of the hospitalized child, as well as the disruption of the parent-child relationship, are the most stressful for parents and are equally stressful for both mothers and fathers. Parents are forced to experience role revision, from the parent of a previously well child to one of a child who is critically ill (Jay \& Youngblut). The inability to hold, comfort, or protect a child from painful procedures may contribute to increased anxiety and lead to a sense of inadequacy as a parent. The exposure to multiple stressors encountered by parents during the critical hospitalization of a child may increase the number of perceived needs that must be promptly identified and addressed.

Several research studies have been conducted to ascertain the perceived needs of family members of critically ill adults (Dockter et al., 1988; Freichels, 1991; Kleinpell \& Powers, 1992; Mathis, 1984; Molter, 1979; Rodgers, 1983); however, exploration of parental needs in the critical care pediatric population is limited (Fisher, 1994; Kasper \& Nyamathi, 1988; Kirschbaum, 1990). Molter (1979) conducted an exploratory, descriptive research study to identify the needs of relatives of critically ill patients, the order of need importance, and need fulfillment. The findings of the study indicated family members could identify their needs and that the priority needs identified included honest, accurate information from caring personnel. The most important need identified was the need for hope. These results were replicated by other researchers in various adult patient populations
(Dockter et al.; Freichels; Kleinpell \& Powers; Mathis; Rodgers), with a priority placed on patient-related informational needs.

Kasper and Nyamathi (1988) conducted a qualitative, descriptive study to examine parental needs as identified by 15 parents of children who had been in a pediatric intensive care unit (PICU) for 1.5 to 7 days. Data analysis revealed 280 need statements expressed by the parents, which were categorized as psychologic $(58 \%)$, physical $(27 \%)$, and sociologic $(15 \%)$ in origin. The findings also identified 37 specific subcategories of need, with childrelated information being the most frequently cited subcategory of psychologic needs. Seventy-three percent of the parents wanted to be given accurate, honest information in an expedient manner. The most frequently expressed need in the study was to be with the child in the PICU, which was identified by $80 \%$ of the parents. Other priority needs that were identified included the ability to visit frequently and to assist in the care of the child.

Kirschbaum (1990) sought to identify the needs of 41 parents of critically ill children and the perceived importance of these needs. Data were collected within 48 hours of transfer from PICU to the floor. The most important needs identified were to feel there was hope, an assurance of the best care possible was being given to their child, and to know how their child was being treated medically. The findings indicated that other child-related informational needs were important and included knowing specific facts and receiving honest answers about their child's progress, prognosis, and possible changes in condition.

Fisher (1994) compared the needs of 15 mothers and 15 fathers of 30 children hospitalized in the PICU. In this study, the parents ranked $83 \%$ of the need statements as important. According to Fisher, this finding is indicative of the all-consuming thoughts concerning their critically ill child that parents experience. Overall, the mothers ranked the need statements with greater importance than the fathers. Four of the five priority needs identified by mothers and fathers were informational needs, with the fifth need statement being related to hopefulness. 


\section{Perceived Needs of Parents of Critically Ill Children}

\section{Conceptual Framework}

Crisis theory and King's conceptual framework for nursing were used to provide the conceptual underpinnings for this study. King's (1981) conceptual framework for nursing allows for theoretical operation of the concepts of crisis theory as well as a process for application in the clinical arena.

Crisis theory. Crisis theory postulates that when individuals are exposed to certain conditions or stressors in their environment that exceed their abilities to problem solve or cope with those demands, an imbalance or crisis occurs. During this period of disorganization and anxiety, many abortive attempts are made to seek resolution (Caplan, 1971). The effect of the exposure to such conditions or stressors is dependent on the individual's perception of the event.

When exposed to the crisis event, normal coping and defense mechanisms utilized by the family may be inadequate to restore organization and equilibrium. Danger arises when individuals within the family structure are dramatically influenced by prolonged, negative effects of the crisis on their mental and physical well-being (Woolley, 1990). The goal of crisis intervention is to assist individuals toward normalcy. Movement toward normalcy can provide situational control, promote coherence, and restore equilibrium in the family structure. The individuals may learn new coping mechanisms that will assist them when exposed to other crises in the future (Woolley).

King's conceptual framework. King's conceptual framework for nursing (1981) consists of three interacting, open systems: (1) individuals as personal systems, (2) two or more individuals forming interpersonal systems, and (3) larger groups, with common interests, forming social systems. According to King, individuals are in constant, dynamic interaction with each other and their environment to maintain health. Interference in the ability to adjust to internal or external stressors, or a change in role function, results in a biological, psychological, or social state of illness.

Concepts integral to King's conceptual framework include interaction, transaction, perception, and control.
These concepts provide a framework to structure the development of holistic family assessments in an interactive process. Interaction and transaction, which rely on interdependence, mutuality, and anticipatory guidance, facilitate the accurate assessment of the parental needs. With accurate assessment of parental needs, the pediatric critical care nurse is able to incorporate the family into the plan of care in order to initiate crisis intervention, stabilization, and parental education. When nursing interventions are based on parental needs as they are perceived by the critical care nurse, wasted energy may be expended by the nurse addressing nonexistent needs or needs already fulfilled by others (Molter, 1979).

During parent-nurse interactions, acknowledgment and verbalization of parental uncertainties and fears can occur. Nurses can utilize transactions to provide parents with the information and education necessary to understand their child's illness and prognosis, the environment, and the roles of healthcare professionals. Information decreases parental uncertainty and fear, resulting in a reduction of stress, which minimizes escalation of the crisis event (Woolley, 1990). Nursing interventions focused on accurately identified needs can lead families to regain organization, equilibrium, and control. This will allow families to learn new coping mechanisms while promoting family coherence and support as hypothesized in crisis theory and King's (1981) conceptual framework for nursing.

\section{Methods}

A descriptive, comparative research design was used to identify the needs of parents of critically ill children admitted to the PICU in a 485-bed midwestern hospital. Data were collected over a 9-month period in an 8-bed PICU.

\section{Sample}

A nonprobability convenience sample consisting of 30 parents of 21 critically ill children and 23 pediatric critical care nurses was recruited for this study. Though the 
interviews were conducted separately to minimize biased responses, data analyses were limited to the responses of the female family member for each critically ill child participating in the study $(n=21)$ and the primary critical care nurses caring for each family $(n=17)$. Four of the nurses participated in the study twice with different families. The parents in this study were primary caregivers (PCGs) of a critically ill child (newborn through 15 years) hospitalized for 36 to 84 hours in the PICU, were 18 years or older, and spoke and understood English. The critically ill children were admitted to the PICU for the first time with an acute onset of an illness or a surgical procedure requiring hemodynamic monitoring, observation or stabilization in a critical care unit.

Primary caregivers were not selected for inclusion in this study if their child died during the hospitalization or if inclusion would compromise a parent's emotional wellbeing. In addition, PCGs of critically ill children admitted as the result of suspected child abuse were excluded, as well as parents of premature infants and children with disorders requiring regular, intermittent hospitalizations.

Pediatric critical care nurses who were currently employed in a pediatric critical care unit in a metropoli$\tan$ Midwestern hospital were included in this study. The majority of the nurses participating in the study had been registered nurses for at least 7 years and held associate degrees in nursing. There were not any critical care nurses with advanced nursing degrees employed in the PICU.

Primary caregiver sample. Nineteen of the PCGs were the mothers $(90.5 \%)$ of a critically ill child in the PICU. The remaining PCGs participating in the study included a grandmother (4.8\%) and an aunt (4.8\%). The age of the PCGs ranged from 19 to 51 years, with a mean of 30.6 years $(S D=8.8)$. Twenty-nine percent of the PCGs were employed full time, while another $48 \%$ were homemakers. The majority of the PCGs had completed high school, with an average of 14.5 years of education $(S D=2.6)$. Fourteen of the PCGs had at least one other child at home. The distance from the hospital ranged from 1 to 90 miles $(M=30.7, S D=22.9)$. Hours of visitation in the PICU ranged from 7 to 24 hours $(M=18.9$,
$S D=5.6)$, and $9(42.9 \%)$ of the PCGs stayed at the hospital 24 hours per day.

The ages of the children ranged from 3 to 168 months (14 years), with a mean age of 47.5 months or about 4 years $(S D=11.0)$. Thirteen of the children had never been hospitalized prior to this admission to the PICU. Eight of the children were admitted for respiratory illnesses. Other diagnoses included emergency surgery (4), complications from scheduled surgery (2), dehydration (1), ingestion of a substance (1), seizures (1), head injuries or injuries received from being in a motor vehicle accident (4).

Nurse sample. The majority of the nurse respondents were female $(94.4 \%)$. The age of the nurse respondents ranged from 24 to 46 years $(M=35.8, S D=7.6)$. Seventytwo percent of the nurses were employed in a full-time position. Approximately $72 \%$ of the nurse respondents had one or more children. While $43 \%$ of the nurse respondents had experienced having an adult family member in a critical care unit, none had ever been a patient in a critical care unit or had experienced having any of their own children in a critical care unit.

\section{Instruments}

The instruments in this study included a demographic data form for parents and pediatric critical care nurses and the Critical Care Family Needs Inventory (CCFNI), modified for pediatrics, and the CCFNI to reflect the pediatric critical care nurses' perception of parental needs.

The CCFNI is a 45-item instrument developed to quantify the significance of perceived family needs (Molter, 1979). The need statements are rated on a 4-point scale ranging from 1 (not important) to 4 (very important). The total score is indicative of the relative importance of needs experienced by family members of critically ill patients.

Kirschbaum (1990) modified the CCFNI for use in the pediatric population by adding eight specific need statements. These need statements were developed from a review of the literature and expert opinion. The eight pediatric need statements that were added to the CCFNI 


\section{Perceived Needs of Parents of Critically Ill Children}

were (1) being recognized as important to my child's recovery, (2) maintaining a sense of family togetherness by having my ill child's siblings visit, (3) having help in supporting my ill child's reaction to the intensive care unit, (4) taking time to maintain the marital relationship with my spouse, (5) having a place to sleep near the intensive care unit, (6) being able to talk to other parents whose child is critically ill or has survived a similar illness, (7) receiving help in responding to the reactions of my ill child's siblings, and (8) protecting myself from sights or procedures that my child is experiencing (Kirschbaum, 1990).

Psychometric evaluation of the CCFNI has been performed in several studies. Leske (1991) replicated Molter's study to evaluate its psychometric properties, including its factor structure. The five-factor solution of the CCFNI subdivided the need statements into five conceptual categories: support, comfort, information, proximity, and assurance. Content validity was established for the eight need statements added to the CCFNI by Kirschbaum (1990). Internal consistency reliability of the CCFNI in previous studies ranged from .90 to .95 (Freichels, 1991; Leske; Mathis, 1984; Rodgers, 1983). In this study, internal consistency reliability of the modified pediatric version of the CCFNI was .94 for the primary caregivers and .93 for the nurses.

\section{Procedure}

Permission to conduct the study was obtained from the Institutional Review Board of the university and the nursing research committee at the clinical site. Current patient census in the pediatric critical care unit was reviewed to ascertain eligibility for inclusion in the study. Primary caregivers were contacted by the researcher or designee, no earlier than 36 hours or later than 84 hours following admission of their child to the critical care unit. Primary caregivers were told the purpose of the study and informed consent was obtained. Data collection occurred between 36 and 76 hours of admission to the PICU, with a mean data collection time at $\mathbf{4 8 . 3}$ hours, using a structured interview technique. Pediatric critical

care nurses assigned to the critically ill child whose PCG participated in the study were contacted separately to obtain their consent for participation in the study. All the nurses participating in the study were interviewed immediately before or after the PCG interview.

\section{Results}

The order of perceived importance for the 53 need statements was determined using measures of central tendency and dispersion. Mean ratings for individual need statements by the PCGs ranged from 2.1 to 4.0 . The PCGs universally identified eight needs as priorities. These included the need to know the expected outcome and the medical treatment; to be given honest answers; to be assured that the best care was being given by caring personnel; to see and visit their child frequently; and to be notified of any changes in the condition of their child. The 10 most important need statements identified by the PCGs are presented in Table 1. In comparison, the

Table 1. Ten Most Important Needs Identified by Primary Caregivers

\section{Need Statement}

- Feel that hospital personnel care

- To see their child frequently

- Called at home about changes in condition

- Assured that the best care is given

- Know how their child is being treated medically

- To visit at any time

- Have questions answered honestly

- To know the expected outcome

- Recognized as important to their child's recovery

- Receive information once a day

$\begin{array}{cc}\begin{array}{c}\text { Very } \\ \text { Important (\%) }\end{array} & \boldsymbol{M}(S D) \\ 100 & 4.0(0.00) \\ 100 & 4.0(0.00) \\ 100 & 4.0(0.00) \\ & \\ 100 & 4.0(0.00) \\ 100 & 4.0(0.00) \\ 100 & 4.0(0.00) \\ 100 & 4.0(0.00) \\ 100 & 4.0(0.00) \\ 95 & 3.95(0.22) \\ 95 & 3.95(0.22)\end{array}$


mean ratings of the need statements by the nurses ranged from 2.6 to 3.9. While the nurses identified similar needs as important, significance was placed on childrelated information and hopefulness. The 10 most important need statements identified by the nurses are presented in Table 2.

The relationship of individual needs identified by PCGs and nurse pairs were analyzed with the Wilcoxon matched-pairs signed-ranks test. There was a significant difference in perceived importance for 10 need statements (Table 3). The PCGs indicated that it was significant to be recognized as important to their child's recovery, to feel that hospital personnel cared about their child, and to talk to the same physician every day. The PCGs also identified having the waiting room near their child and a close place to sleep as significant priorities. Situational support, comfort, and self-care needs were perceived with significantly less importance in the matched pairs by the PCGs than the critical care nurses.

Table 2. Ten Most Important Needs Identified by Critical Care Nurses

Need Statement

- Know how their child is being treated medically

- Receive information once a day

- Feel there is hope

- Assured that the best care is given

- Have questions answered honestly

- To know the expected outcome

- To see their child frequently

- To visit at any time

- Have explanations of the environment before going into the PICU for the first time

- Feel that hospital personnel care

\begin{tabular}{|c|c|}
\hline $\begin{array}{c}\text { Very } \\
\text { Important (\%) }\end{array}$ & $M(\mathrm{SD})$ \\
\hline 95.2 & $3.9(0.44)$ \\
\hline 90.5 & $3.9(0.30)$ \\
\hline 90.5 & $3.9(0.30)$ \\
\hline 90.5 & $3.9(0.30)$ \\
\hline 90.5 & $3.9(0.30)$ \\
\hline 90.5 & $3.9(0.30)$ \\
\hline 85.7 & $3.86(0.36)$ \\
\hline 85.7 & $3.86(0.36)$ \\
\hline 84.2 & $3.86(0.37)$ \\
\hline 81.0 & $3.81(0.40)$ \\
\hline
\end{tabular}

Table 3. Relationship of Individual Needs Identified by PCG/Nurse Pairs

\section{Need Statement}

- Feel that hospital personnel care about their child

- Talk to same doctor every day

- Recognized as important to their child's recovery

- Having the waiting room near their child

- Place to sleep near the PICU

- Having explanations of the environment before going into the PICU for the first time

- Assured that it is all right to leave the hospital

- Talk to the same nurse every day

- Having another person with them when visiting the PICU

- Having someone concerned with their health

${ }^{*} p<.10 ;{ }^{* *} p<.05 ;{ }^{* *} p<.01$

\section{Number of Ranks Nurse < Parent (Mean Rank)}

4 (2.5)

$5(3.0)$

$5(3.0)$

7 (5.3)

$8(5.6)$

$0(0.0)$

$2(4.5)$

$4(6.0)$

$3(4.0)$

$2(8.0)$

\section{Number of Ranks \\ Nurse > Parent \\ (Mean Rank)}

$0(0.0)$

$0(0.0)$

$0(0.0)$

$2(4.0)$

$2(5.0)$

$9(5.0)$

$8(5.8)$

$10(8.1)$

$9(7.3)$

$13(8.0)$
Z

$-1.82^{*}$

$-2.02^{* *}$

$-2.02^{* *}$

$-1.72^{*}$

$-1.78^{*}$

$-2.66^{* * *}$

$-1.88^{*}$

$-1.79^{*}$

$-2.12^{* *}$

$-2.49 * *$ 


\section{Perceived Needs of Parents of Critically Ill Children}

A paired $t$ test was used to compare the PCG need statement total score $(M=181.25, S D=19.1)$ with the critical care nurse total score $(M=185.31, S D=18.5)$ computed from the CCFNI. Even though there were differences in the order of individual need statements, there was not a significant difference between the two groups in the overall perception of family needs $(t(15)=0.77, p=.46)$.

\section{Discussion}

In general, the PCGs and the nurses identified similar needs as important. Both groups perceived that information, assurance, and proximity needs were more important than support and comfort needs. These findings are consistent with previous research (Fisher, 1994; Freichels, 1991; Kasper \& Nyamathi, 1988; Kirschbaum, 1990; Molter, 1979). Furthermore, the PCGs placed emphasis on needs concerning child-related information and caring personnel. Other priority needs identified were to receive information at least once a day, to see their critically ill child frequently, and to visit at any time. These findings demonstrate how important it is for PCGs to maintain close contact with their critically ill child.

Even though a significant emphasis was placed on informational needs by both the PCGs and the nurses, there were differences between the two groups related to the source of information. The need to talk with the doctor every day was identified by $95 \%$ of the PCGs as very important, while only $14.3 \%$ identified the need to talk to the same nurse every day as important. In contrast, the nurses felt it was slightly more important to talk to the same nurse every day ( $24 \%$ ). Although the nurses perceived communicating with the doctor daily as highly important $(71 \%)$, it was perceived with less emphasis than by the PCGs. These findings are consistent with a traditional healthcare perspective, which views the physician as the sole source of information (Kleinpell, 1991). However, critical care nurses spend more time with PCGs than any other member of the healthcare team and are in an optimal position to provide important child-related information and anticipatory guidance to families.
Being recognized as important to their child's recovery was identified as very important by $95.2 \%$ of the parents compared with $71.4 \%$ of the nurses. The recognition of how important the PCG is to their child's recovery may facilitate fulfillment of the need to regain loss of control and assurance while adjusting to PCG role revision experienced during the unexpected critical hospitalization. In addition, as parental roles become altered during the critical hospitalization, PCGs may attempt to maintain the parental role function of protection through presence, vigilance, and validation that hospital personnel care about their child both physically and emotionally. The nurses may perceive that assistance in the care of the critically ill child serves as an intervention to facilitate recognition of the parents as important in the child's recovery, as well as to maintain parental roles. This seems to be of less concern to parents at this critical time. While both these need statements result in the same outcome for parental involvement in the child's care, the differences in how needs are perceived could precipitate a conflict between the parent and nurse dyad.

Needs related to their own health were considered less important by PCGs than the physiological and psychological needs of their critically ill child. Only $14.3 \%$ of the PCGs perceived their own health as a priority need. The low priority placed on self-care needs may indicate the inability of PCGs to focus attention on themselves during their child's critical hospitalization. Spiritual needs were perceived as the least important need among both groups. Affirmation of spiritual needs may be indicative of feelings of despair. In addition, denial of the severity of their child's illness may result in a low priority placed on spiritual needs. Regardless of the low priority placed on spiritual needs, $52.4 \%$ of the PCGs and $57.1 \%$ of the nurses identified the need to verbalize about the possible death of the child as very important.

While the critical care nurses accurately identified parental needs, the magnitude of the importance as perceived by the PCGs was not recognized. There were eight needs identified by all the PCGs as very important; however, none of the needs were perceived as universally important by the nurse respondents. Moreover, the 
PCGs identified the need to feel that hospital personnel care about their child with paramount importance. Yet, only $81 \%$ of the nurses considered the need for caring professionals as a priority among the PCGs. In conjunction, there was a significant difference between the PCG and nurse matched pairs in their perception of the need for caring professionals. This discrepancy between the PCGs and the nurses raises an empirical question: What do PCGs of critically ill children and nurses perceive as demonstrations of caring behaviors? In a qualitative study conducted by Burfitt, Greiner, Miers, Kinney, and Branyon (1993), following transfer from a critical care unit, patients described caring as attentive, vigilant behavior demonstrated by nurses. Components of vigilant behavior included technological expertise, nurturance and doing "something extra or unexpected." Yet the question remains as to what are the extra or unexpected behaviors performed by nurses that these patients and their family members perceive as carative. The identification and understanding of PCGs' perception of carative behaviors is essential to nursing practice and its related foci.

\section{Conclusions}

Information, assurance, and proximity to the critically ill child were identified as priority needs of the parents in this study. These needs are best identified by critical care nurses who have interactive roles with parents and their children. By consistently incorporating these perceived needs identified by the parents into the plan of care, critical care nurses can assist the parents in the identification and fulfillment of support, comfort, and self-care needs that have less perceived importance.

The importance of PCGs and their roles in the recovery of a critically ill child must be recognized by nurses. Nurses must develop and initiate strategies that will enhance parental participation and decision making regarding the care of their child. The inclusion and active participation of PCGs in the care of the child will facilitate movement toward regaining parental control, adaptation (LaMontagne et al., 1995), and crisis resolution.
Further inquiry in this area by nurse researchers is warranted, as well as investigation of specific nursing interventions designed to assist family members to adapt to the critical illness of one of their children. In addition, a family-centered empirical focus is necessary to understand the complex needs of PCGs and children as a whole, not as separate entities (Rennick, 1995). Nurse clinicians, educators, administrators, and researchers collectively can contribute to the existing body of nursing knowledge to facilitate a more holistic approach to meeting both the needs of our patients and their families.

Acknowledgment. The author acknowledges Andrea Bostrom, PhD, RN, Associate Professor, Grand Valley State University, Allendale, MI, for her encouragement, guidance, and counsel during the research process.

\section{References}

Burfitt, S., Greiner, D., Miers, L., Kinney, M., \& Branyon, M. (1993). Professional nurse caring as perceived by critically ill patients: A phenomenologic study. American Journal of Critical Care, 2, 489-499.

Caplan, G. (1971). An approach to community health. New York: Grune \& Stratton.

Carter, M., Miles, M., Buford, T., \& Hassanein, R. (1985). Parental environmental stress in pediatric intensive care units. Dimensions in Critical Care Nursing, 4, 180-188.

Dockter, B., Black, D., Hovell, M., Engleberg, D., Amick, T., Neimier, D., $\&$ Sheets, N. (1988). Families and intensive care nurses: Comparisons of perceptions. Patient Education $\mathcal{E}$ Counseling, 12, 29-36.

Fisher, M. (1994). Identified needs of parents in a pediatric intensive care unit. Critical Care Nurse, 14, 82-90.

Freichels, T. (1991). Needs of family members of patients in the intensive care unit over time. Critical Care Nursing Quarterly, 14, 16-29.

Graves, C., \& Hayes, V. (1996). Do nurses and parents of children with chronic conditions agree on parental needs? Journal of Pediatric Nursing, 11, 288-299.

Heuer, L. (1993). Parental stressors in a pediatric intensive care unit. Pediatric Nursing, 19, 128-131. 


\section{Perceived Needs of Parents of Critically Ill Children}

Jay, S., \& Youngblut, J. (1991). Parental stress associated with pediatric critical care nursing: Linking research and practice. AACN Clinical Issues in Critical Care Nursing, 2, 276-284.

Kasper, J., \& Nyamathi, A. (1988). Parents of children in the pediatric intensive care unit: What are their needs? Heart $\mathcal{E}$ Lung, 17, 574-581.

King, I. (1981). A theory for nursing: System, concepts, process. New York: Delmar.

Kirschbaum, M. (1990). Needs of parents of critically ill children. Dimensions in Critical Care Nursing, 9, 344-352.

Kleinpell, R. (1991). Needs of families of critically ill patients: A literature review. Critical Care Nurse, 11, 34-40.

Kleinpell, R., \& Powers, M. (1992). Needs of family members of intensive care unit patients. Applied Nursing Research, 5, 2-8.

LaMontagne, L., Johnson, B., \& Hepworth, J. (1995). Evolution of parental stress and coping processes: A framework for critical care practice. Journal of Pediatric Nursing, 10, 212-218.

LaMontagne, L., \& Pawlak, R. (1990). Stress and coping of parents of children in a pediatric intensive care unit. Heart $\mathcal{E}$ Lung, 19, $416-421$.

Leske, J. (1991). Internal psychometric properties of the Critical Care Family Needs Inventory. Heart E Lung, 20, 236-244.

Mathis, M. (1984). Personal needs of family members of critically ill patients with and without acute brain injury. Journal of Neurosurgical Nursing, 16, 36-44.

Miles, M., Carter, M., Spicher, C., \& Hassanein, R. (1984). Maternal and paternal stress reactions when a child is hospitalized in a pediatric intensive care unit. Issues in Comprehensive Pediatric Nursing, 7, $333-342$.

Mishel, M. (1983). Parents' perception of uncertainty concerning their hospitalized child. Nursing Research, 32, 324-330.

Molter, N. (1979). Needs of relatives of critically ill patients: A descriptive study. Heart $\mathcal{E}$ Lung, 8, 332-339.

Philichi, L. (1989). Family adaptation during a pediatric intensive care hospitalization. Joumal of Pediatric Nursing, 4, 268-276.

Rennick, J. (1995). The changing profile of an acute childhood illness: A need for the development of family nursing knowledge. Journal of Adzanced Nursing, 22, 258-266.
Rodgers, M. (1983). Needs of relatives of cardiac surgery patients during the critical care phase. Focus on Critical Care, 10, 50-55.

Tomlinson, P., Kirschbaum, M., Harbaugh, B., \& Anderson, K. (1996). The influence of illness severity and family resources on maternal uncertainty during critical pediatric hospitalization. American Journal of Critical Care, 5, 140-146.

Turner, M., Tomlinson, P., \& Harbaugh, B. (1990). Parental uncertainty in critical care hospitalization of children. Maternal-Child Nursing Journal, 19, 45-62.

Woolley, N. (1990). Crisis theory: A paradigm of effective intervention with families of critically ill people. Journal of Adtanced Nursing, 15, 1402-1408.

Author contact: Linda D. Scott 6263 Plainfield Avenue

Kalamazoo, Michigan 49004

ldscott@umich.edu

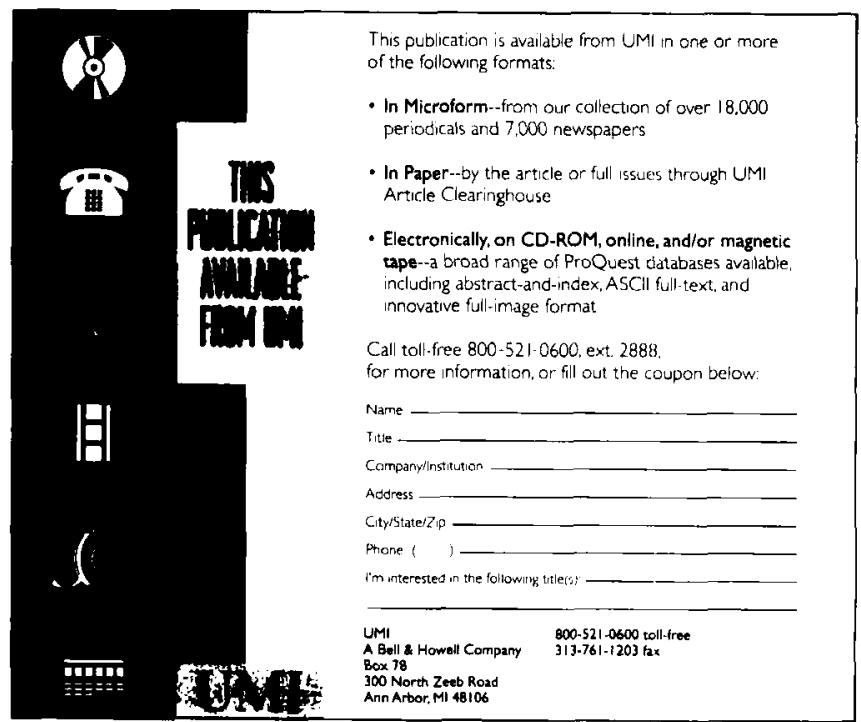

